\title{
CONTROLE DA ANTRACNOSE EM MAMÃO POR EXTRATOS VEGETAIS
}

\author{
César Luis Siqueira Junior \\ Doutor em Biociências e Biotecnologia/UENF/RJ \\ Laboratório Integrado de Biologia Vegetal/UNIRIO/RJ \\ cesarjunior.unirio@gmail.com
}

Thais de Castro Moraes

Aluna de Iniciação Científica do curso de Bacharelado em Ciências Biológicas/Laboratório Integrado de Biologia Vegetal/UNIRIO/RJ

t.moraes90@gmail.com

\section{Jade Ayres Barbedo Martins}

Aluna de Iniciação Científica do curso de Bacharelado em Ciências Biológicas/Laboratório Integrado de Biologia Vegetal/UNIRIO/RJ

jade_abm@yahoo.com.br

\section{Maria das Graças Machado Freire}

Doutora em Biologia funcional e molecular/UNICAMP/SP

Laboratório de Química e Biomoléculas/ISECENSA/RJ

maria.freire@terra.com.br

\section{RESUMO}

Para avaliar o efeito de extratos de plantas sobre o crescimento de Colletotrichum gloeosporioides, extratos etanólicos de sementes de pitomba, folhas de tanchagem, boldo e espada-de-são-jorge, foram obtidos e incorporados em meio contendo batata-dextrose-agar (BDA) nas concentrações de $1 \%, 5 \%$ e $10 \%$ (v/v). O crescimento micelial do fungo foi avaliado nesse meio. Os resultados demonstraram o efeito inibitório dos extratos de pitomba e tanchagem aumentam consideravelmente em concentrações maiores que $5 \%(\mathrm{v} / \mathrm{v})$, levando a uma inibição quase total do fungo ( $90 \%$ e $100 \%$, respectivamente). Em contraste, os extratos de boldo e espada-de-são-jorge, apresentaram baixo potencial inibitório mesmo na concentração de $10 \%$ (v/v) causando a redução de apenas $8 \%$ e $23 \%$, respectivamente, no crescimento fúngico.

A atividade fungitóxica detectada nos extratos testados nesse trabalho evidencia o potencial uso dos mesmos como biopreservadores de frutos de mamão, prevenindo a contaminação e a consequente perda dos frutos no período pós-colheita.

Palavras chave:doença, atividade antifúngica, biopreservadores, compostos secundários

\begin{abstract}
To evaluate the effect of plant extracts on the growth of Colletotrichum gloeosporioides, ethanolic extracts from pitomba seeds, plantain, sword-of-saint-george and maigoya leaves were obtained and incorporated in potato-dextrose-agar medium (PDA) at concentrations of $1 \%, 5 \%$ and $10 \%(\mathrm{v} / \mathrm{v})$. Micelial growth was evaluated in these media. The results demonstrated the inhibitory effect of extracts from plantain and pitomba increase significantly at concentrations above $5 \%(\mathrm{v} / \mathrm{v})$, causing almost complete inhibition of the fungal growth $(90 \%$ and $100 \%$, respectively). In contrast, maigoya and sword-of-saint-george extracts showed small inhibition even at a concentration of $10 \%(\mathrm{v} / \mathrm{v})$, causing a reduction, in the fungal growth, of only $8 \%$ and $23 \%$, respectively. The fungitoxic activity detected in the extracts tested in this work show the potential usage as biopreservers of papaya, preventing contamination and consequent loss of fruits during postharvest.
\end{abstract}


Keywords:disease, antifungal activity, biopreservers, secondary compounds 


\section{INTRODUÇ̃̃O}

O Brasil é um dos maiores produtores e exportadores mundiais de mamão, contribuindo com $25 \%$ da produção mundial em 2002 (FNP, 2005). O mamão é um fruto muito perecível após seu amadurecimento, podendo ser mantido por até três semanas sob condições adequadas de estocagem (Kader, 1992). Um dos fatores que podem influenciar a qualidade pós-colheita do fruto de mamão é a ocorrência de doenças fúngicas. O fungo Colletotrichum gloeosporioides, agente causador da antracnose, é um dos patógenos mais importantes de frutos de mamão no período de pós-colheita, causando perdas de até $90 \%$ de frutos em estações favoráveis à doença (Tatagiba et al, 2002). Sua infecção inicia-se ainda na floração, mas permanece latente até o amadurecimento e colheita do fruto, quando então coloniza os tecidos desenvolvendo os subseqüentes sintomas da antracnose, que geralmente aparecem durante o transporte e comercialização (Snowdon, 1990; Dickman e Alvarez, 1983; Serra et al, 2008). Em adição, as infecções no período póscolheita se tornam mais fáceis devido à presença de inóculos fúngicos no ambiente de armazenamento como também pela considerável quantidade de ferimentos provocados no fruto após a colheita (Capdeville et al, 2007).

A aplicação de fungicidas durante a formação do fruto é uma das maneiras mais efetivas de controle da doença (Liberato e Zambolim, 2002). Contudo, o controle de doenças fúngicas com fungicidas tem sido considerado um problema visto que alguns tratamentos afetam o processo de amadurecimento natural dos frutos e além disso, o uso permanente de fungicidas pode levar ao aparecimento de variações resistentes do fungo (Gamagae et al, 2003).

Formas alternativas de controle têm sido empregadas visando a biopreservação de frutos. Tratamentos físicos envolvendo irradiação com raios gama e ultravioleta-C (UV-C $254 \mathrm{~nm}$ ) vêm sendo empregados por exibirem efeitos fungicidas e também por induzirem a resistência em frutos (Wilson et al, 1994; Cia et al, 2007). Cia et al. (2007) mostraram que a irradiação com raios gama contribuem para a redução de perdas pós colheita causadas pela infecção pelo fungo C. gloeosporioides inibindo a germinação e o crescimento micelial. Outra forma de controle alternativo é a aplicação de biocontrole a partir da associação de organismos vivos com compostos químicos. O potencial fungicida de bicarbonato de sódio combinado a Candida oleophila foi demonstrado por reduzir a incidência e severidade da antracnose em frutos em condições de armazenamento (Gamagae et al, 2004).

A utilização de compostos naturais de plantas vem se mostrando uma forma alternativa de tratamento com potencial no controle de doenças pós- colheita. Alguns estudos, por exemplo, tem apontado para a possibilidade de aplicação de óleos essenciais de plantas no controle de doenças causadas por microorganismos patogênicos em plantas (Lo Cantore et al, 2004; Burt, 2004; Iacobellis et al, 2005). Ribeiro e Bedendo (1999) demonstraram que extratos aquosos de alho, menta, pimenta e mamona exibiram efeitos inibitórios sobre o fungo C. gloeosporioides quando testados in vitro causando redução da produção de conídios ou inibindo o crescimento micelial. Mais recentemente Jun-Young et al. (2006) demonstraram que compostos ativos presentes no extrato metanólico de açafrão (Ccurma longa) inibem efetivamente o crescimento micelial de espécies do gênero Colletotrichum, incluindo o C. gloeosporioides. Diante do exposto, o presente trabalho foi desenvolvido com o objetivo de verificar o potencial de extratos etanólicos de sementes de pitomba (Talisia esculenta), folhas de boldo (Plectranthus barbatus), tanchagem (Plantago major) e espada-de-são-jorge (Sansevieria trifasciata L.) em inibir o crescimento micelial in vitro do patógeno C. gloeosporioides, causador da antracnose em frutos de mamão.

\section{MATERIAIS E MÉTODOS}

\subsection{Obtenção do patógeno}

Cepas do fungo fitopatogênico Colletotrichum gloeosporioides foram cedidas gentilmente pela Dra. Maria Lígia Rodrigues Macedo (Universidade Federal de Mato Grosso do Sul - UFMS).

\subsection{Obtenção dos extratos vegetais}

Para esse trabalho foram utilizadas sementes de pitomba (Talisia esculenta), folhas de boldo (Plectranthus barbatus), tanchagem (Plantago major) e espada-de-são-jorge (Sansevieria trifasciata L.) As plantas foram coletadas na região sudeste do Brasil, no município de Campos dos Goytacazes, no Estado do 
Rio de Janeiro, com exceção das sementes de pitomba que foram cedidas pela Dra. Maria Lígia Rodrigues Macedo (Universidade Federal de Mato Grosso do Sul - UFMS). Após a coleta as plantas foram levadas para o laboratório, onde foram lavadas em água corrente e colocadas em solução de hipoclorito a 10\% por 20 minutos, priorizando a eliminação de microorganismos presentes na superfície. Em seguida material foi lavado (individualmente) em água destilada para a retirada do excesso de hipoclorito e mantido em repouso por 24 horas, para redução da umidade. Após o período, o material foi acondicionado em sacos de papel e mantido em estufa à $45^{\circ} \mathrm{C}$ durante $48 \mathrm{~h}$. Os extratos vegetais foram produzidos a partir de uma modificação da metodologia descrita por Ribeiro e Bedendo (1999). Resumidamente, o material seco foi triturado em etanol por 3 min em liquidificador. O homogenato resultante da trituração foi filtrado em papel de filtro e o extrato bruto líquido foi concentrado em rotaevaporador, obtendo-se extrato etanólico.

\subsection{Avaliação da atividade antifúngica dos extratos}

A atividade antifúngica dos extratos vegetais foi analisada por um procedimento baseado na metodologia descrita por Ribeiro e Bedendo (1999). Os extratos, individualmente, foram adicionados à placa de Petri contendo meio BDA (batata $200 \mathrm{~g} / \mathrm{L}$, dextrose $20 \mathrm{~g} / \mathrm{L}$, ágar $15 \mathrm{~g} / \mathrm{L}$ ) à aproximadamente $45^{\circ} \mathrm{C}$, em concentrações crescentes de $1 \%, 5 \%$ e $10 \%(\mathrm{v} / \mathrm{v})$ onde cada concentração representou um tratamento. Placas contendo somente BDA serviram como controle. Após a solidificação, um inóculo de $5 \mathrm{~mm}$ de diâmetro do micélio de $C$. gloeosporioides, com 10 dias de idade, foi repicado para o centro de cada placa, as quais foram vedadas com filme PVC e mantidas a $25^{\circ} \mathrm{C}$, com fotoperíodo de $12 \mathrm{~h}$ luz e $12 \mathrm{~h}$ escuro, até que as amostras controle atingissem $2 / 3$ do tamanho da placa. A avaliação do crescimento micelial foi verificada pela mensuração do diâmetro das colônias, obtida pela média de três medidas diametralmente opostas.

O delineamento experimental utilizado foi inteiramente casualizado, constituído de 13 tratamentos, com três repetições, cujos fatores foram 4 extratos, 3 concentrações $(1 \%, 5 \%$ e $10 \%)$ e o controle.

\section{RESULTADOS E DISCUSSÃO}

Na figura 1 observa-se que, das plantas testadas, apenas duas apresentam efeito inibitório acentuado sobre o crescimento do fungo, in vitro. Quando adicionado ao meio BDA $(5 \% \mathrm{v} / \mathrm{v})$, o extrato de sementes de pitomba inibiu significativamente o crescimento micelial do fungo atingindo a inibição média de $80 \%$, quando comparados ao controle. A adição do extrato de tanchagem, na mesma concentração, também causou uma inibição efetiva do crescimento fúngico ( $65 \%)$. A inibição causada por esses dois extratos se eleva quando a concentração dos mesmos é aumentada no meio de cultura, sugerindo uma inibição dosagem dependente. À concentração de $10 \%$ (v/v) o extrato de pitomba interrompe o crescimento micelial do fungo C. gloeosporioides, enquanto que o extrato de tanchagem provoca uma inibição de cerca de $90 \%$. Essa forte inibição no crescimento do fungo provocada pelo uso de extratos vegetais já foi observada por outros grupos de pesquisa. Rozwalka (2003) observou uma inibição total do crescimento in vitro do C. gloeosporioides quando extrato de flores de cravo-da-índia foi adicionado ao meio BDA, na mesma concentração utilizada em nosso trabalho $(10 \%(\mathrm{v} / \mathrm{v}))$. 


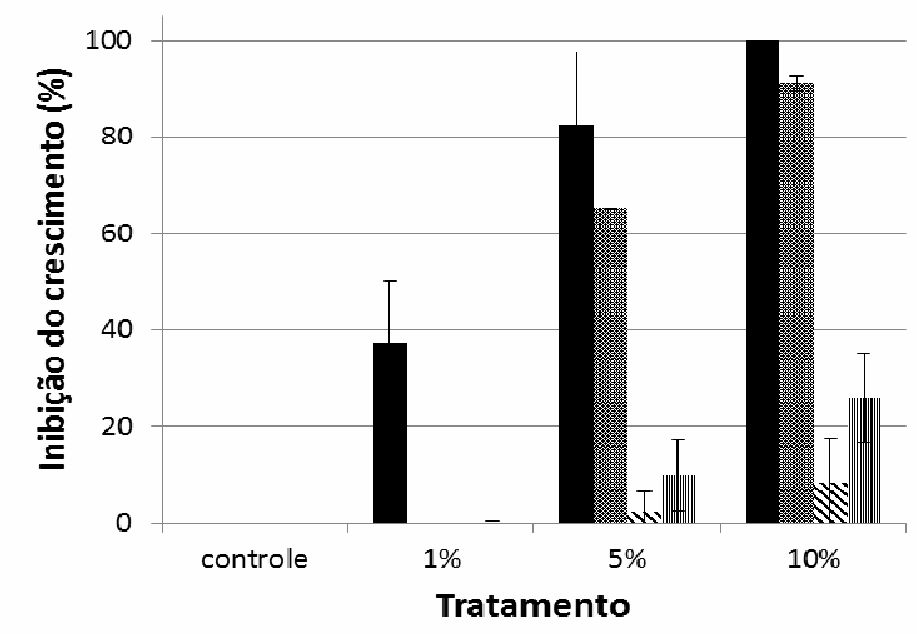

pitomba

路 tanchagem

s゙ boldo

IIIII espada-de-são -

jorge

Figura 1 - Porcentagem de inibição do crescimento micelial de Colletotrichum gloeosporioides in vitro em meio BDA contendo extratos etanólicos de plantas em diferentes concentrações. As barras representam os valores médios $( \pm \mathrm{DP})$ dos dados obtidos em três experimentos independentes.

O fungo $C$. gloeosporioides aparentemente não apresenta sensibilidade a todos os extratos vegetais utilizados, como pode ser observado na figura 1. Os extratos de folhas de boldo e de espada-de-são-jorge, mesmo em concentrações maiores $(10 \% \mathrm{v} / \mathrm{v})$ não apresentam efeito expressivo sobre o crescimento micelial do fungo, in vitro. Esses resultados são corroborados com análises anteriores descritas por Jun-Young et al.(2006) que avaliando o potencial de curcuminóides isolados de açafrão-da-terra (Curcuma longa) contra o crescimento do mesmo fungo, demonstraram que o $C$. gloeosporioides não apresenta sensibilidade a esses compostos, quando comparados aos fungos do mesmo gênero, C. acutatum e C. coccodes. Em adição, Ribeiro e Bedendo (1999) também demonstraram que extratos aquosos de alho, hortelã, mamona e pimenta apresentam efeito parcial na inibição do fungo $C$ gloeosporioides, mesmo em concentrações maiores. Milanesi et al. (2009) demonstraram o mesmo fungo só apresenta susceptibilidade parcial ao extrato de carqueja (Baccharis trimera) dentre as plantas utilizadas no trabalho: pitangueira (Eugenia uniflora), cinamomo (Melia azeradach), louro (Laurus nobilis) e cancorosa (Jodina rhombifolia). Esses dados indicam que inibição relativa provocada por extratos vegetais não inviabilizam sua aplicação como controle alternativo contra a antracnose, uma vez que sugerem a existência de compostos secundários biologicamente ativos capazes de exercer atividade antifúngica sobre o patógeno Colletotrichum gloeosporioides, nas plantas utilizadas. Processos que permitam a purificação desses compostos poderiam levar ao isolamento das substâncias ativas contra o fungo.

É importante salientar que os resultados obtidos nesse trabalho permitem uma aplicação prática e barata para agricultores. Informações sobre o preparo de extratos e o potencial dos mesmos contra o desenvolvimento da doença possibilitaria, no futuro, a produção caseira de extratos vegetais para utilização como método alternativo no controle da antracnose de frutos de mamoeiro.

\section{CONCLUSÃO}

Os extratos etanólicos de sementes de pitomba e de folhas de tanchagem apresentam potencial fungicida contra o fungo causador de antracnose em frutos de mamoeiro, Colletotrichum gloeosporioides. Dessa forma essas plantas poderiam ser empregadas como forma alternativa de controle de doenças em culturas de mamoeiro.

\section{AGRADECIMENTOS}

Os autores agradecem a $\mathrm{Dr}^{\mathrm{a}}$ Maria Ligia R. Macedo por ceder gentilmente a Cepa do fungo utilizado nesse trabalho. Essa pesquisa foi financiada pela FAPERJ, UNIRIO e ISECENSA. 


\section{REFERÊNCIAS BIBLIOGRÁFICAS}

BURT, S. Essential oils: their antibacterial properties and potential applications in foods - a review. International Journal of Food Microbiology V. 94, p. 223-253, 2004.

CAPDEVILLE, G.; SOUZA JR, M. T.; SANTOS, J. R. P; MIRANDA, S. P.; CAETANO, A. R.; TORRES, F. A. G. Selection and testing of epiphytic yeasts to control anthracnose in post-harvest of papaya fruit. Scientia Horticulturae. v. 111, p. 179-185, 2007.

CIA, P.; PASCHOLATI, S. F.; BENATO, E. A.; CAMILI, E. C.; SANTOS, C. A. Effects of gamma and UV-C irradiation on the postharvest control of papaya anthracnose. Postharvest Biology and Technology. V. 43, p. 366-373, 2007.

DICKMAN, M. B.; ALVAREZ, A. M. Latent infection of papaya caused by Colletotrichum gloeosporioides Plant Disease. v. 67, p.748-750, 1983.

FNP. Consultoria \& AgroInformativos, 2005. Anuário da Agricultura Brasileira, São Paulo, 2005.

GAMAGAE, S. U.; SIVAKUMAR, D.; WILSON WIJERATNAM, R. S., WIJESUNDERA, R. L. C. Use of sodium bicarbonate and Candida oleophila to control anthracnose in papaya during storage. Crop Protection. V. 22, p. $775-779,2003$

GAMAGAE, S. U.; SIVAKUMARA, D.; WIJESUNDERAB, R. L. C. Evaluation of post-harvest application of sodium bicarbonate incorporated wax formulation and Candida oleophila for the control of anthracnose of papaya. Crop Protection. v. 23, p. 575-579, 2004.

IACOBELLIS, N. S.; LO CANTORE, P.; CAPASSO, F.; SENATORE, F. Antibacterial Activity of Cuminum cyminum L. and Carum carvi L. Essential Oils. Journal of Agricultural and Food Chemistry. v. 53, p. 57-61, 2005.

JUN-YOUNG, C.; CHO, G. J.; LEE, S.; JANG, K. S.; LIM, H. K.; LIM, C. H.; LEE, S. O.; CHO, K. Y.; KIM, J. C. Antifungal activity against Colletotrichum spp. of curcuminoids isolated from Curcuma longa L. rhizomes. Journal of Microbiology and Biotechnology. v. 16, p. 280-285, 2006.

KADER, A.A. Postharvest Technology of Horticultural Crops. Division of Agriculture and Natural Resources, Oakland. 1992.

LIBERATO, J.R. \& ZAMBOLIM, L. Controle das doenças causadas por fungos, bactérias e fitonematóides em mamoeiro. In ZAMBOLIM, L.; VALE, F. X. R.; MONTEIRO, A. J. A.; COSTA H. Controle de doenças de plantas fruteiras. v.2. Viçosa: Universidade Federal de Viçosa. p. 1023-1169. 2002.

LO CANTORE, P.; IACOBELLIS, N. S.; DE MARCO, A.; CAPASSO, F.; SENATORE, F. Antibacterial Activity of Coriandrum sativum L. and Foeniculum vulgare Miller Var. vulgare (Miller) Essential Oils. Journal of Agricultural and Food Chemistry. v. 52, p. 7862-7866, 2004.

MILANESI, P. M.; BLUME, E.; MUNIZ, M. F. B.; BRAND, S. C.; JUNGES, E.; MANZONI, C. G.; WEBER, M. N. D. Ação fungitóxica de extratos vegetais sobre o crescimento micelial de Colletotrichum gloeosporioides. Revista da FZVA. V. 16, p. 01-13, 2009.

RIBEIRO, L. F.; BEDENDO, I. P. Efeito inibitório de extratos vegetais sobre Colletotrichum gloeosporioides - agente causal da podridão de frutos de mamoeiro. Scientia Agricola. v. 56, p.1267-1271, 1999.

ROSWALKA, L. C. Controle alternativo da antracnose em frutos de goiabeira, em laboratório. 2003. Tese de Mestrado. 50p. Setor de Ciências Agrárias, Universidade Federal do Paraná. 
SNOWDON, A.L. Color atlas of post-harvest diseases and disorders of fruit and vegetables. Boca Raton: Editora CRC Press, 1990.

SERRA, I.M.R.S.; COELHO, R.S.B.; MENEZES, M. Caracterização fisiológica, patogênica e análise isoenzimática de isolados monospóricos e multispóricos de Colletotrichum gloeosporioides. Summa Phytopathology. V. 34, p.113-120, 2008.

TATAGIBA, J. S.; LIBERATO, J. R.; ZAMBOLIM, L.; VENTURA, J. A.; COSTA, H. Controle e condições climáticas favoráveis à antracnose (Colletotrichum gloeosporioides) do mamoeiro. Fitopatologia Brasileira. V. 27, p. 186-192, 2002.

WILSON, C. L.; EL-GHAOUTH, A.; CHALUTZ, E.; DROBY, S.; STEVENS, C.; LU, J. Y.; KHAN, V.; ARUL, J. Potential of induced resistance to control postharvest disease of fruits and vegetables. Plant Disease. v. 78, p. 837-84, 1994. 